\title{
A Single-Chip UHF RFID Reader Transceiver IC*
}

\author{
Runxi Zhang, Chunqi Shi, Zongsheng Lai \\ Institute of Microelectronic Circuits and Systems, East China Normal University, Shanghai, China \\ Email: rxzhang@ee.ecnu.edu.cn
}

Received April 2013

\begin{abstract}
A single-chip UHF RFID reader transceiver IC has been implemented in $0.18 \mu \mathrm{m}$ SiGe BiCMOS technology. The chip includes all transceiver blocks as RX/TX RF front-end, RX/TX analog baseband, frequency synthesizer and $\mathrm{I}^{2} \mathrm{C}$ with fully-compliant China 800/900 MHz RFID draft, ISO/IEC 18000-6C protocol and ETSI 302 208-1 local regulation. The normal mode receiver in the presence of $-3 \mathrm{dBm}$ self-jammer achieves $-75 \mathrm{dBm} 1 \%$ PER sensitivity. The linear class-A PA integrated in transmitter has $25 \mathrm{dBm}$ OP1 dB output power for CW. The fully-integrated fractional-N frequency synthesizer is designed based on MASH 1-1-1 sigma-delta modulator and $1.8 \mathrm{GHz}$ fundamental frequency LC-VCO for lower in-band and out-of-band phase noise. The measured phase noise is up to $-106 \mathrm{dBc} / \mathrm{Hz} @ 200 \mathrm{kHz}$ and $-131 \mathrm{dBc} / \mathrm{Hz} @ 1 \mathrm{MHz}$ offset from center frequency and the integrated RMS jitter from $10 \mathrm{kHz}$ to $10 \mathrm{MHz}$ is less than $1.6 \mathrm{pS}$. The chip dissipates $330 \mathrm{~mA}$ from $3.3 \mathrm{~V}$ power supply when transmitting $22.4 \mathrm{dBm}$ CW signal and the PAE of linear PA is up to $26 \%$. The chip die area is $16.8 \mathrm{~mm}^{2}$.
\end{abstract}

Keywords: Integrated Circuit; Reader Transceiver; Single-Chip; UHF RFID

\section{Introduction}

UHF RFID reader constructed by discrete components has achieved major market in many applications such as logistic, traffic distribution, asset management and industrial flow line controlling using non-battery-powered system [1]. With the rapid growth of mobile internet service, we hope UHF RFID readers can be integrated into portable devices such as smartphone, PDA and tablet PC. UHF RFID systems use low data rate less than $640 \mathrm{kbps}$ and simple coding schemes like FM0 and miller [2], the digital baseband signal processing can easily be realized using popular ARM-based processor. The integration scheme of UHF RFID reader transceiver is becoming the critical problem and attracting lots of research attention from all over the world [3-6,12].

This paper describes a monolithic UHF RFID reader transceiver for mobile RFID application, which is fabricated in the mature and low-cost $0.18 \mu \mathrm{m}$ SiGe BiCMOS process. The chip includes all transceiver blocks as lownoise and linear RX/TX RF front-end, configurable RX/ TX analog baseband, sigma-delta modulator (SDM) fractional-N frequency synthesizer, linear Class-A PA and some necessary serial interface. The integrated UHF RFID reader is compliant with China 800/900 MHz RF-

*This paper is funded by National Science \& Technology Major Projects of China under Grant 2009ZX01034-002-002-001and East China Normal University Young Scholar Innovation Fund under Grant 78210082.
ID draft [7], ISO/IEC 18000-6C protocol [8] and ETSI 302 208-1 local regulation [9]. In LBT mode, the RX sensitivity is $-85 \mathrm{dBm}$; in normal mode, while the inband self-jammer of supplying energy to passive tag is $-3 \mathrm{dBm}$, the $1 \%$ PER RX sensitivity is about $-75 \mathrm{dBm}$. The reader transmitter sufficiently meets the transmission mask of China draft and ISO/IEC 18000-6C multipleinterrogator mode and the out-of-emission requirements from ETSI 302 208-1 regulation. This paper is organized as follows. In Section 2, we will discuss the reader transceiver system architecture. The circuit implementation is described in Section 3. Section 4 will review measurement results. Finally, we will give a conclusion in Section 5 .

\section{System Architecture}

Figure 1 shows a block diagram of the proposed UHF RFID reader. Through adding small amount of discrete components as RF circulator, antenna, TCXO, protocol controller and data convertor, the reader can realize identification of passive tags. Because some components such as TCXO, data convertor and controller can be shared with smartphones, the transceiver can easily be adopted for those mobile devices with UHF RFID functions. The RX RF front-end operates in 840 to $960 \mathrm{MHz}$ and performs frequency down-conversion. The TX front-end realizes the frequency up-conversion with the help of digital 
domain Hilbert filter and supports three regulated modulations of DSB-, SSB- and PR-ASK for dedicated applications. The RX and TX baseband realizes filtration and application of the desired signal. The SDM fractional-N frequency synthesizer supply clean LO carrier for frequency translation. The integrated PA is used to amplify signal to the maximum allowed power for saving external PA and reducing cost. The high-linearity of PA is helpful in reducing spectrum regrowth in transmitter and meeting transmission mask requirements. The basic chip setting including channel bandwidth selection, operation frequency calibration, DC offset removal and band gap trimming are all configured through I2C interface.

\section{Circuit Implementation}

\subsection{Receiver}

Because the backscattered signal from tags using FM0 or

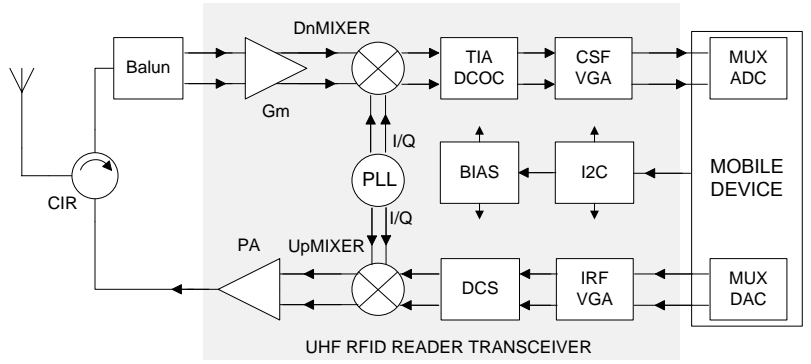

Figure 1. Block diagram of the proposed UHF RFID reader.
Miller and containing no DC component, the I/Q direct-conversion architecture is adopted in RX path. The I/Q architecture is also helpful in coupling with zero phenomenon of amplitude-modulated signal [3]. The most challenging problem with UHF RFID reader design is the unwanted CW leakage from TX to RX in order to energize passive tags. Accompanying with this problem, the receiver must face the correlated amplitude (AM) noise and phase (PM) noise [10]. Assuming the transmitted power is up to $20 \mathrm{dBm}$ [7], the isolation of circulator (or directional coupler) is up to $22 \mathrm{~dB}$ [11], the CW leakage will be $0 \mathrm{dBm}$ level or more $[3,4]$. For various applications, people mainly adopt two methods of cancellation [12] and accommodation [3-6] to deal with it. In comparison, the former needs more auxiliary digital algorithms and RF power consumption, more likely to be used by fixed readers, the latter is usually adopted by mobile readers. Figure 2 shows the proposed front-end schematic consisting of transconductance, switching stage and transimpedance (TIA). The linearity of RF front-end is very important to accommodate the un-wanted while necessary leakage signal, so low current-efficiency and high output impedance transconductor stage and currentmode passive down-mixer is adopted in RF front-end. The small input impedance of TIA assures the low voltage swing before and after switching stage. Because the backscatter signal power is always located around DC, especially for low down-link rate, the lower input-referred noise is a critical design target for high-sensitivity

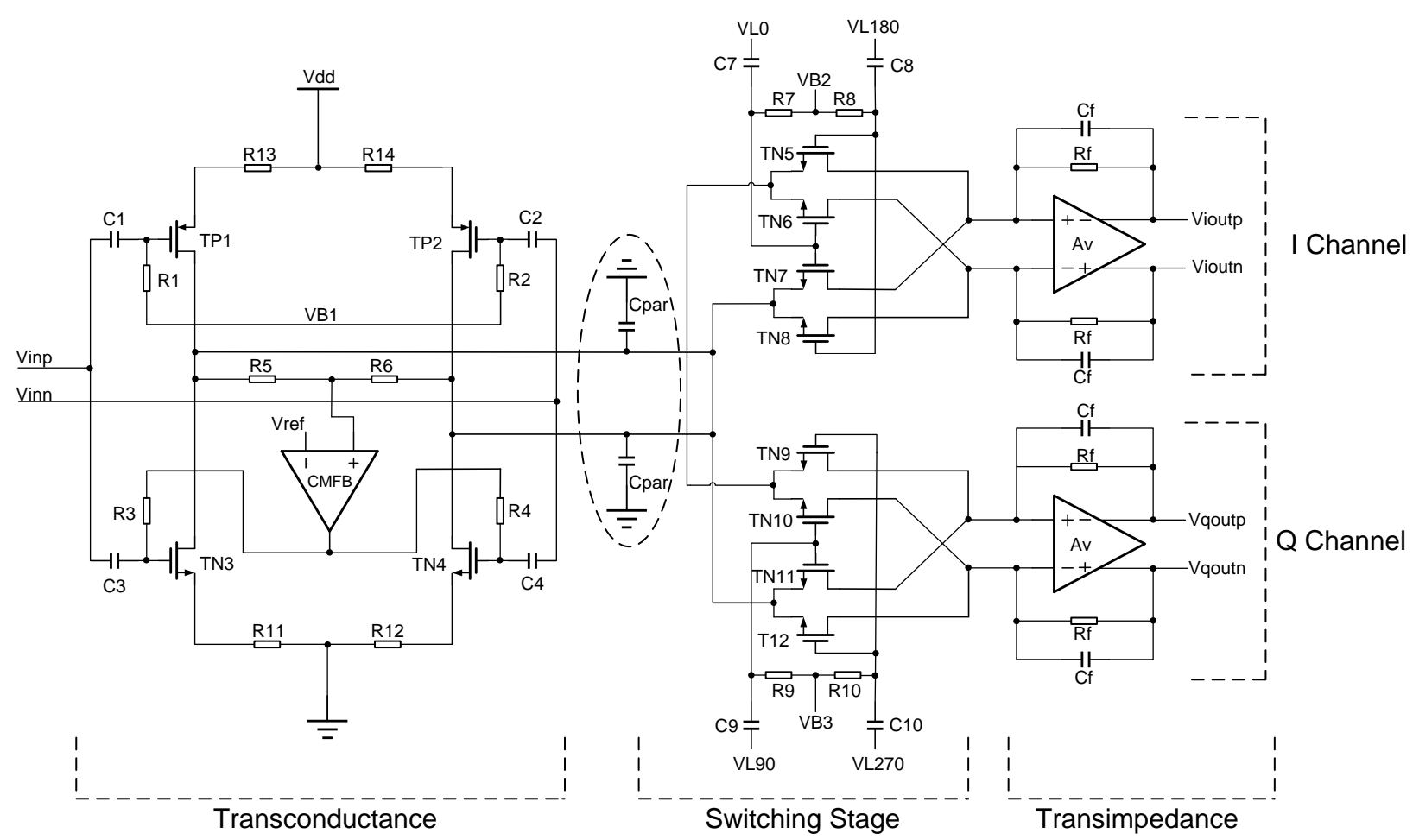

Figure 2. Schematic of the proposed RX RF front-end. 
reader. In addition to AM and PM noise from transmitter, the self-noise of RX RF front-end is contributed by transconductance and operational amplifier (OP-amp) of TIA. We shall trade-off between noise, linearity and conversion gain of RF front-end to select appropriate transconductance. We use low flicker noise lateral PNP transistors as input devices of OP-amp for better noise performance. In fact, the input-referred noise contributed by OP-amp is also related with the switching stage parasitic capacitance, the size of switching transistors should be chosen carefully. The designed RF front-end features a SSB-NF of $18 \mathrm{~dB}$, input P1dB of $6 \mathrm{dBm}$ and conversion gain of $10 \mathrm{~dB}$. In order or to be immune from PM noise of TX leakage, we take same signal source with TX leakage as receiver $\mathrm{LO}$.

Due to different transmit power and TX-RX isolation, the down-mixer will produce sub-1V DC-offset and saturate following stage. The RX analog baseband is preceded by a DC-offset removal circuit which is designed with fast DC-offset cancellation progress and low receive SNR deterioration. A $8^{\text {th }}$-order Butterworth type activeRC filter provides channel selection and amplification with 4 - $60 \mathrm{~dB}$ gain range and $10 \mathrm{~dB}$ gain step and 0.25 1.35 MHz variable bandwidth. The filter cut-off frequency is calibrated by automatic frequency tuning (AFT) circuit for PVT variation. The adopted AFT scheme is illustrated in Figure 3, it will share precise 19.2 MHz with external TCXO. The AFT accuracy is up to $3.2 \%$ and the calibration time is less than $3 \mathrm{uS}$.

\subsection{Transmitter}

The transmitter uses Cartesian direct up-conversion architecture for DSB-, SSB- and PR-ASK modulations. It is comprised of TX variable gain amplifier, anti-aliasing low-pass filter, DC shifter circuit, up-mixer and on-chip linear PA. The analog signal is filtered by I/Q $6^{\text {th }}$-order active-RC filter to attenuate DAC output image and sampling clock noise. The filter output noise should be reduced as low as possible because it will be up-converted to carrier frequency in transmitter and deteriorate receiver noise floor through TX to RX leakage. The TX variable gain stage ranged from $-14 \mathrm{~dB}$ to $10 \mathrm{~dB}$ with $1 \mathrm{~dB}$ step is used to fine-tune TX baseband output amplitude for ACPR performance of transmitter and be fit for variant DAC output. According to [2], the reader should support $80 \%$ - 90\% modulation depth for various applications, a DC shifter circuit without affecting desired signal is introduced between analog baseband and up-mixer. The circuit can supply up to $0.5 \mathrm{~V}$ DC difference in differential line and implement the required modulation depth for DSB-ASK. A linear Gilbert-based I/Q doublebalanced up-mixer with DC-independent load and cur-

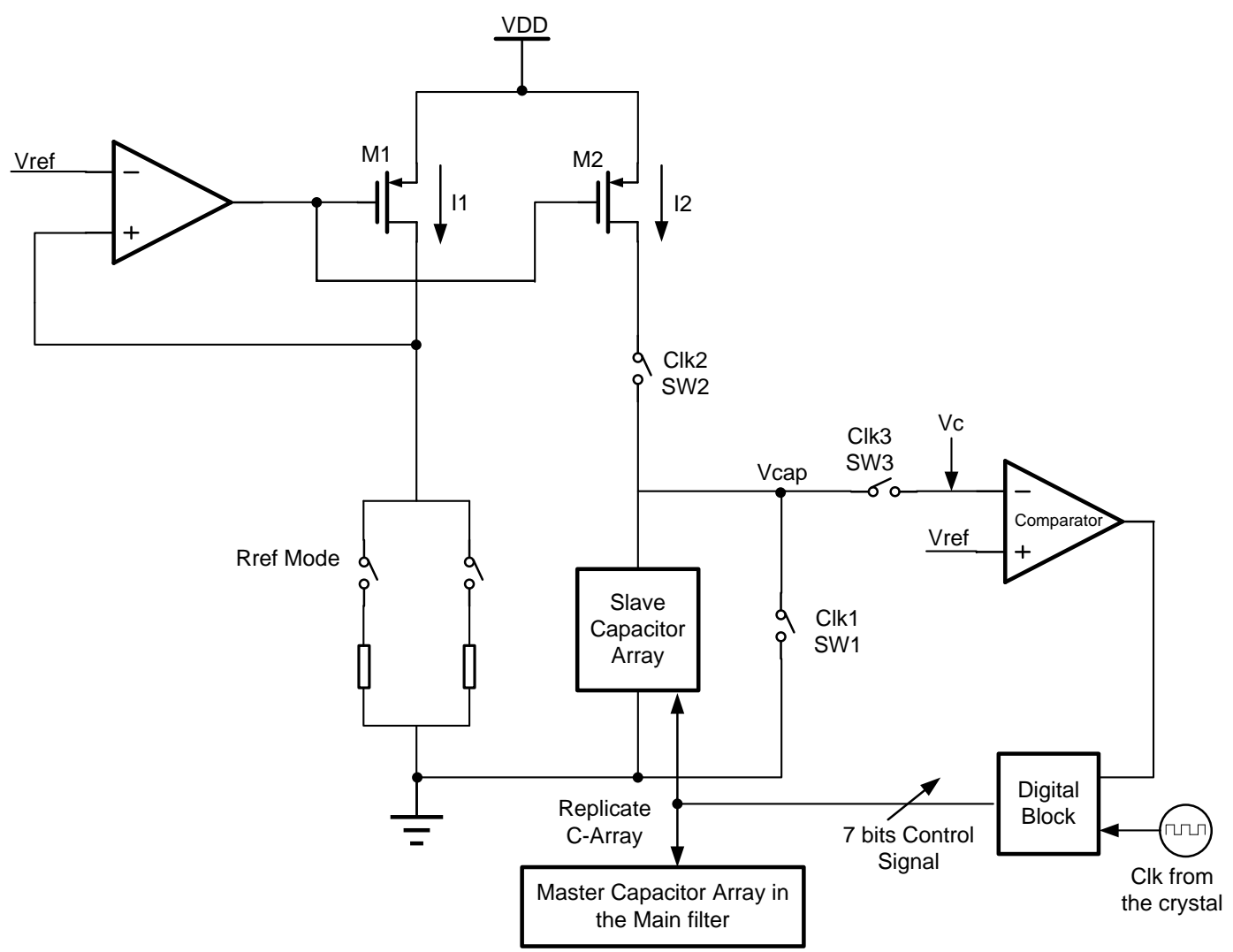

Figure 3. Scheme of AFT adopted in RX filter. 
rentbleeding transistors is adopted to take the baseband output signal. As shown in Figure 4, the switchable inphase path combining with DC shifter circuit can imple- ment the regulated SSB-ASK and PR-ASK. The designed up-mixer has a conversion gain of $0 \mathrm{~dB}$ and output $\mathrm{P} 1 \mathrm{~dB}$ of $3 \mathrm{dBm}$. Figure 5 shows a schematic of the class-APA

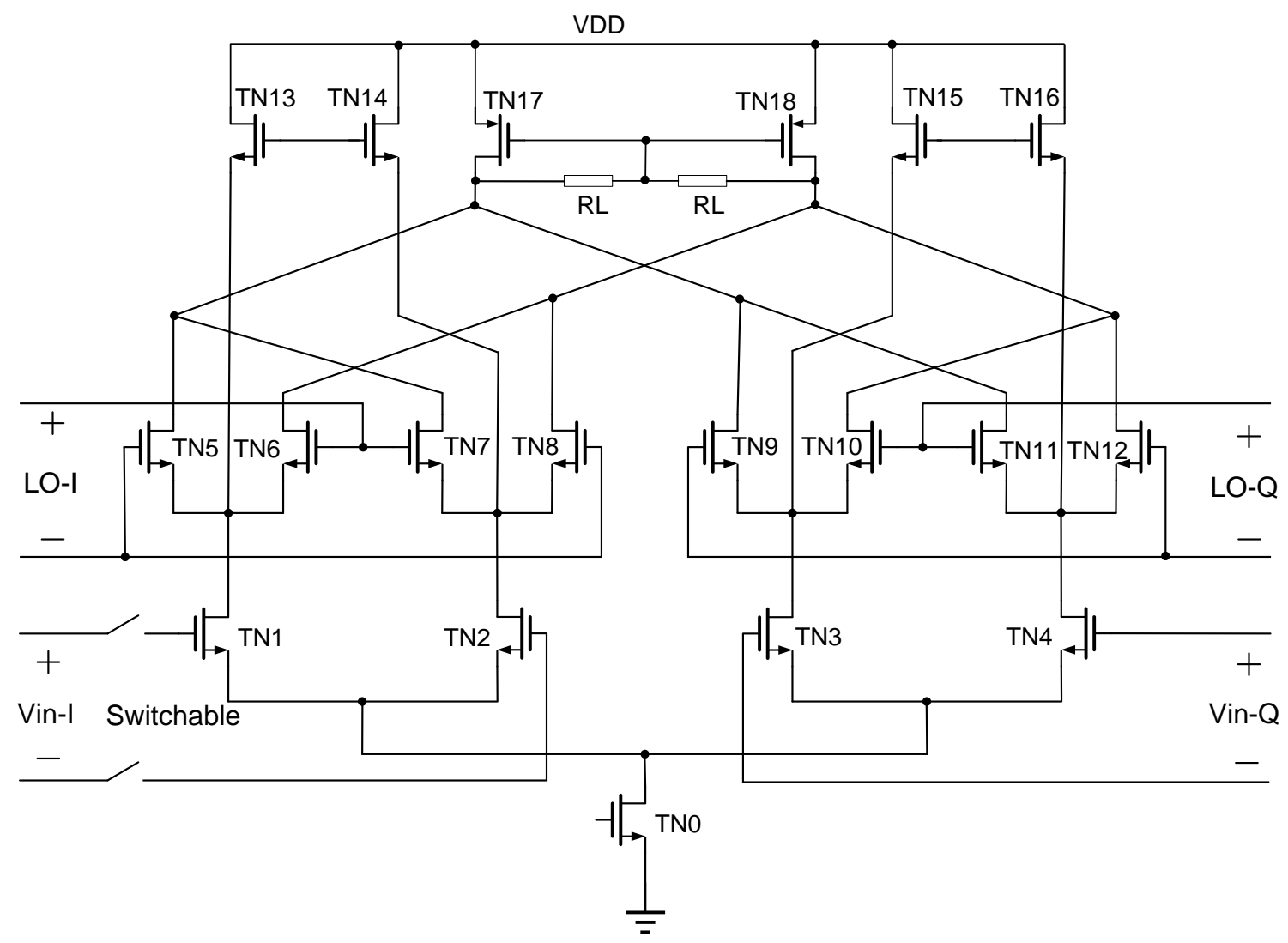

Figure 4. Gilbert-based switchable I/Q upmixer.

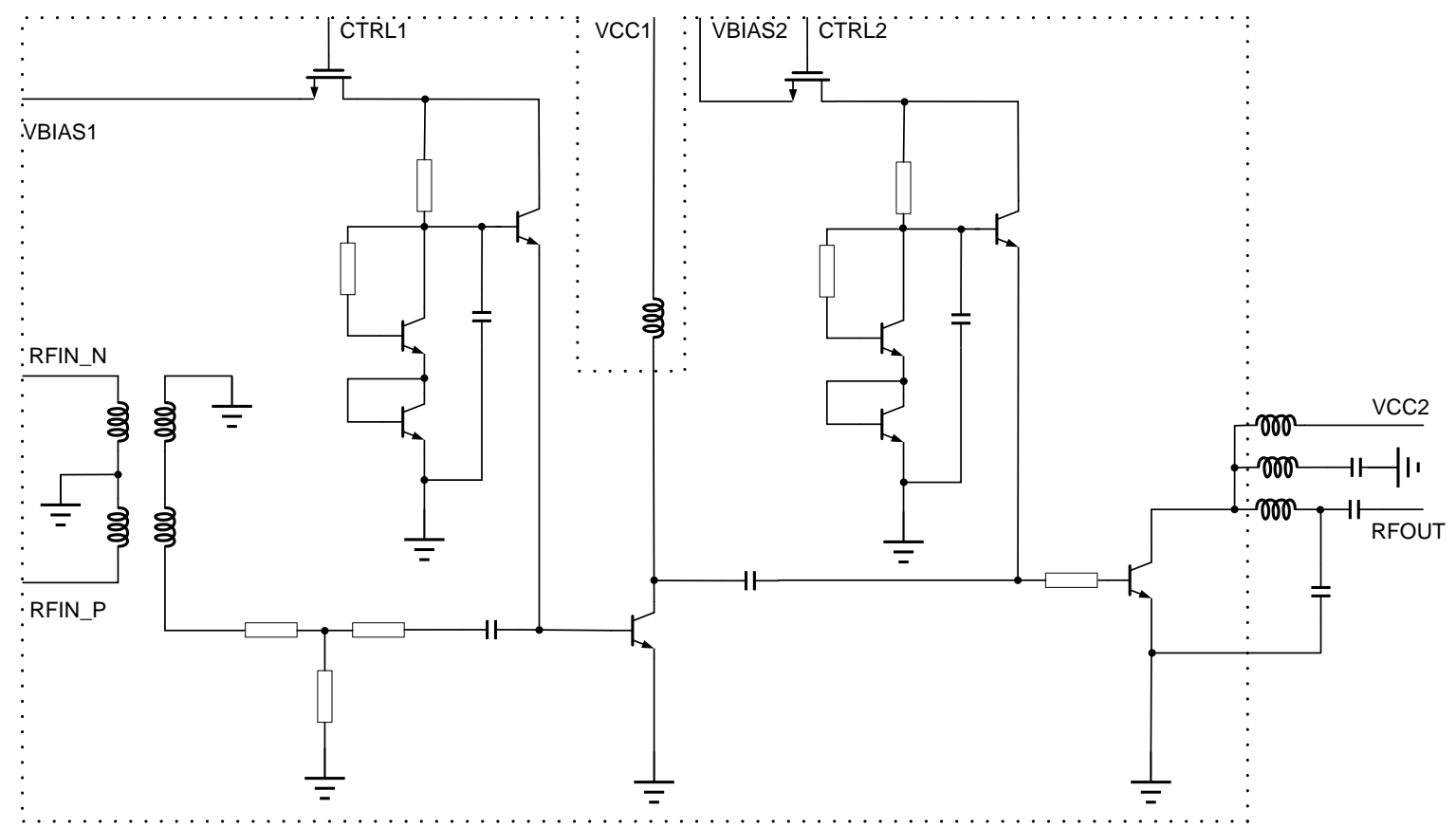

Figure 5. Linear class-A power amplifier. 
with differential input and single-ended output. The integrated balun supplies $-5 \mathrm{~dB}$ attenuation for assuring stable linear operation. A $10.16 \mu \mathrm{m} \times 0.9 \mu \mathrm{m} \times 128$ vertical SiGe NPN with optimized rectangle-type layout acts as output power-cell. The PA using double-bondpad features a $25 \mathrm{dBm}$ OP1dB with 37\% PAE from $3.3 \mathrm{~V}$ power supply. The deep-nwell is used to isolate on-chip PA with other circuits on die.

\subsection{Frequency Synthesizer}

The SDM fractional-N frequency synthesizer shown in Figure 6 integrates all blocks including PFD, CP, 1.8 $\mathrm{GHz}$ constant $\mathrm{k}_{\mathrm{VCO}}$ double-switch LC-VCO with capacitor and varactor array, 8/9 dual-modulus divider, $\mathrm{P} / \mathrm{S}$ counter, 20-bit $3^{\text {rd }}$-order MASH 1-1-1 SDM and I/Q prescaler except for loop filter components. An AFC circuit with 4-bit output is included for automatic sub-band selection in PLL while the tuning time is about $23 \mathrm{uS}$. The LC- VCO using 2.5 V LDO supply has frequency range from 1.44 to $2.18 \mathrm{GHz}$ and FOM of -186.8 .

\section{Measurement Results}

The single-chip UHF RFID reader transceiver IC is implemented $0.18 \mu \mathrm{m}$ SiGe BiCMOS process, the die photograph is shown in Figure 7. The die size is $16.8 \mathrm{~mm}^{2}$ and packaged into QFN64. All signal paths are differen- tial to be immune from coupling noise. Figure 8 shows the measured phase noise performance from specified VCO test pins, the carrier phase noise will be $6 \mathrm{~dB}$ lower. The spot phase noise is $-106 /-130 \mathrm{dBc} / \mathrm{Hz}$ at $200 \mathrm{kHz} /$ $1 \mathrm{MHz}$ offset from center frequency. The RMS jitter from $10 \mathrm{kHz}$ to $10 \mathrm{MHz}$ is less than $1.6 \mathrm{pS}$. When transmitting $22.4 \mathrm{dBm}$ and $80 \mathrm{kbps}$ modulated signal, the transmission power is $-45 \mathrm{dBc}$ at adjacent channel and $-58.2 \mathrm{dBc}$ at alternative channel, it sufficiently meets the transmission mask for multiple-interrogator environments. The measured sensitivity of receiver with $1 \%$ PER criterion is $-75 \mathrm{dBm}$ in the presence of $-3 \mathrm{dBm}$ leakage at RX input port. The chip dissipates $330 \mathrm{~mA}$ from $3.3 \mathrm{~V}$ power supply when transmitting $22.4 \mathrm{dBm}$ CW. Table 1 compares the measured performance with some state-of-theart counterparts.

\section{Conclusion}

According to the demand of portable RFID, a single-chip UHF RFID reader is presented in this paper. It integrates all building blocks of transceiver and is fully compliant with China draft, ISO/IEC 18000-6C protocol and ETSI 302 208-1 regulation. In normal mode, it consumes $1.1 \mathrm{~W}$ when transmitting a $22.4 \mathrm{dBm} \mathrm{CW}$ signal and sufficiently meets the multiple-interrogator ACPR requirement. With a few added discrete components as directional

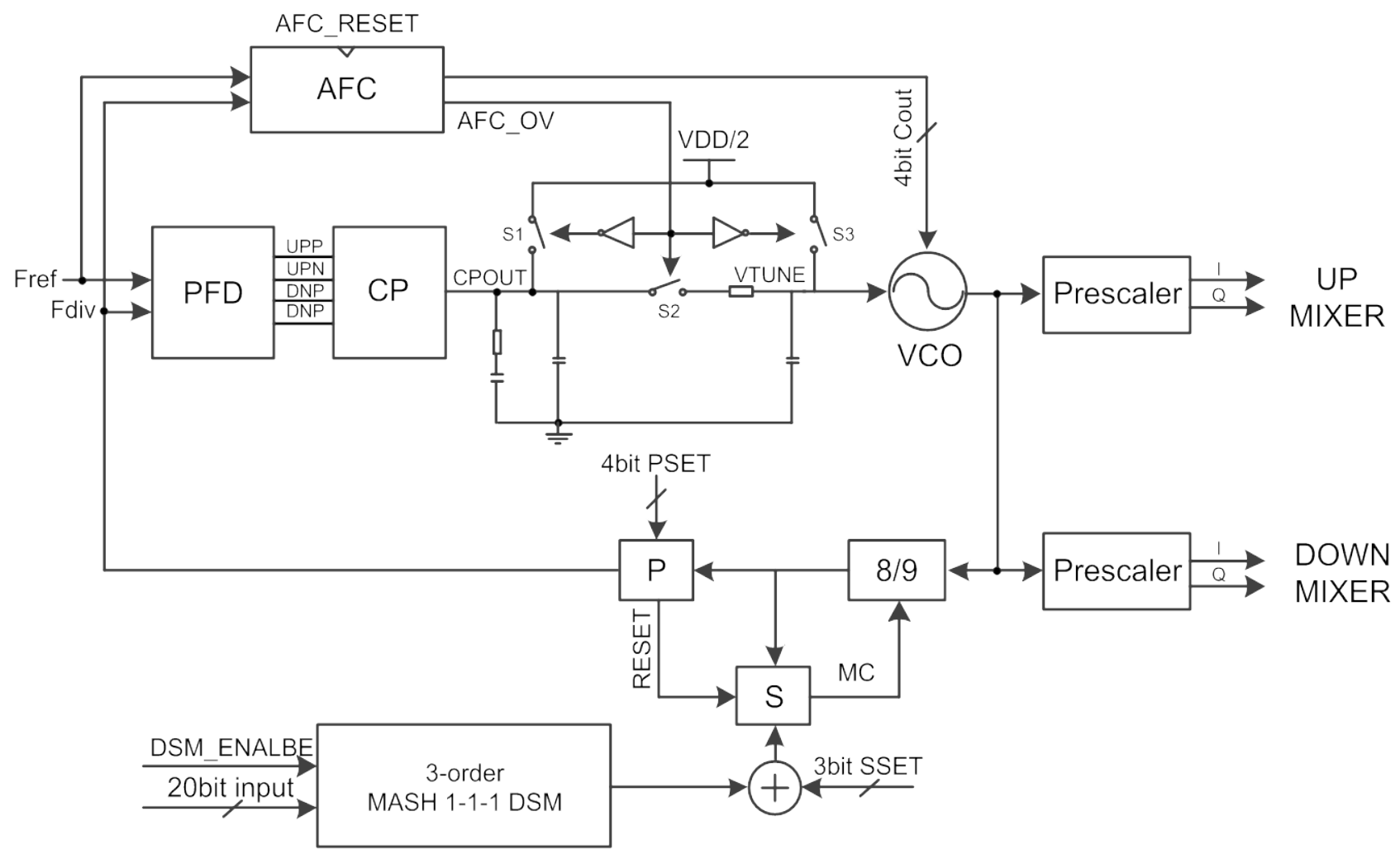

Figure 6. System diagram of fractional-N PLL. 


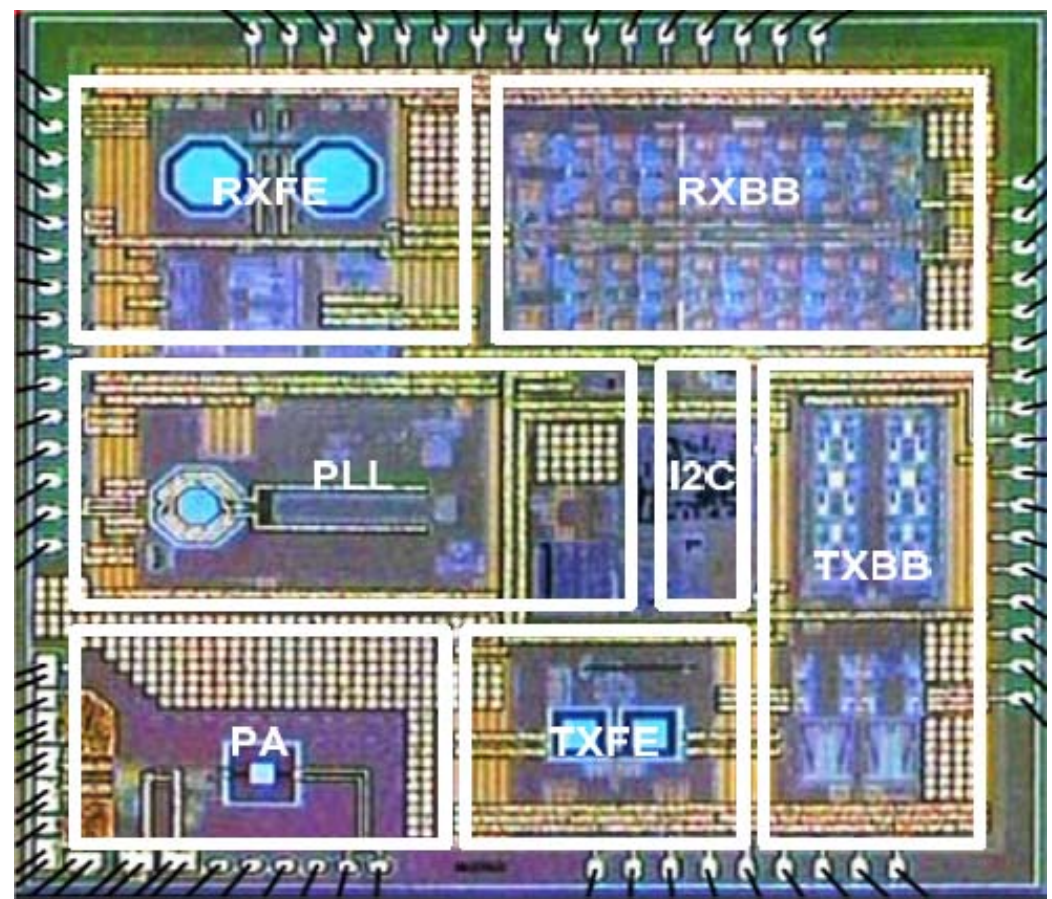

Figure 7. Chip microphotograph.

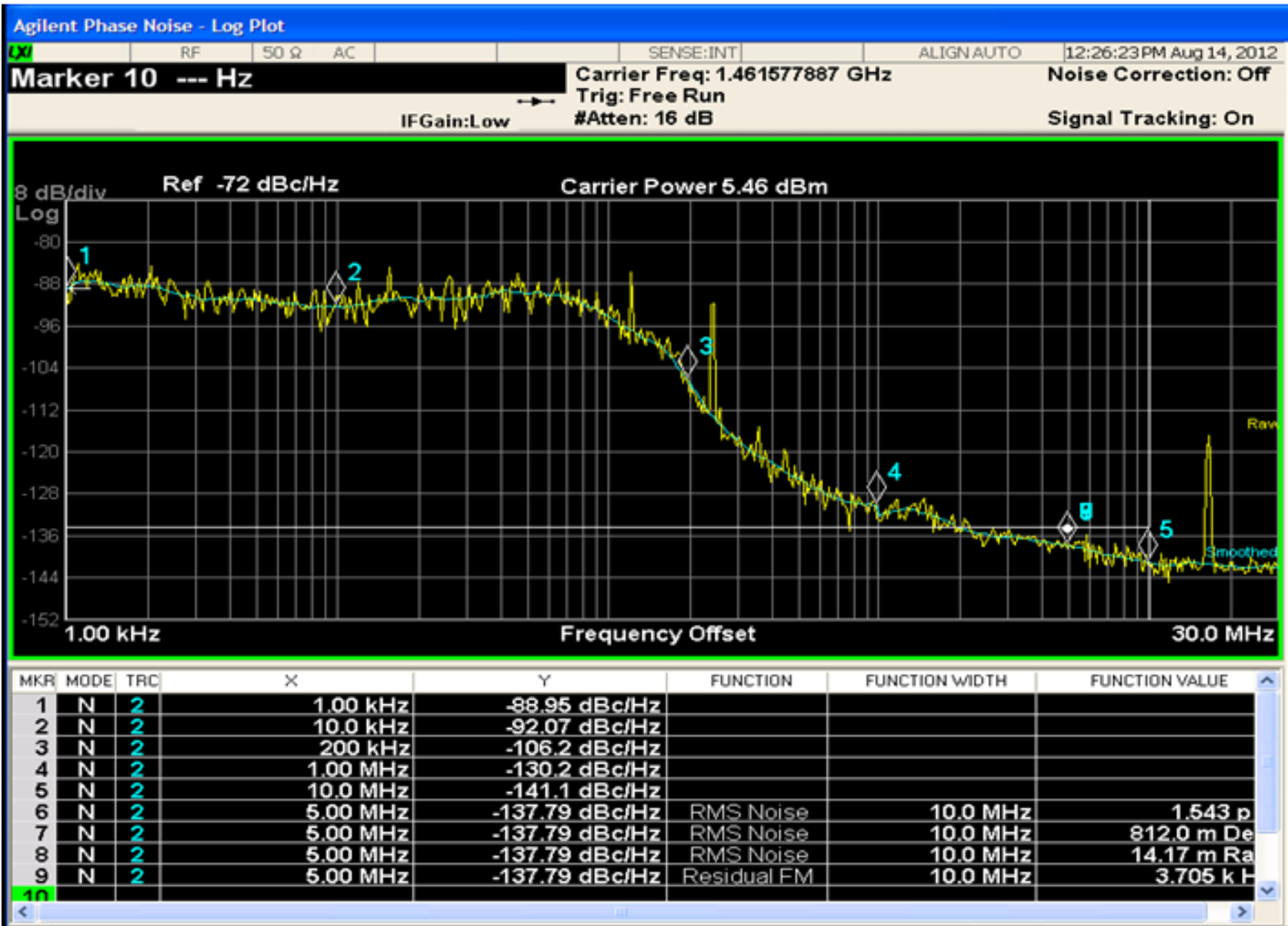

Figure 8. Measured phase noise performance. 
Table 1. Performance comparison of the existed UHF RFID reader transceiver.

\begin{tabular}{|c|c|c|c|c|c|}
\hline Reference & [3] & [4] & [5] & [6] & This Work \\
\hline Process & $0.18 \mu \mathrm{m}$ BiCMOS & $0.18 \mu \mathrm{m}$ CMOS & $0.18 \mu \mathrm{m}$ CMOS & $0.18 \mu \mathrm{m}$ CMOS & $0.18 \mu \mathrm{m}$ BiCMOS \\
\hline Protocol & $\begin{array}{c}\text { EPC C1G2 } \\
\text { ISO 18000-6C }\end{array}$ & $\begin{array}{c}\text { EPC C1G2 } \\
\text { ISO 18000-6C }\end{array}$ & $\begin{array}{c}\text { EPC C1G2 } \\
\text { ISO 18000-6C }\end{array}$ & $\begin{array}{c}\text { EPC C1G2 } \\
\text { ISO 18000-6C }\end{array}$ & $\begin{array}{c}\text { EPC C1G2 } \\
\text { ISO 18000-6C }\end{array}$ \\
\hline Frequency Range & $860-960 \mathrm{MHz}$ & $860-960 \mathrm{MHz}$ & $840-925 \mathrm{MHz}$ & $840-960 \mathrm{MHz}$ & $840-960 \mathrm{MHz}$ \\
\hline Sensitivity@1\% PER & -73 dBm@2 dBm SJ & -70 dBm@-5 dBm SJ & -79 dBm@-13 dBm SJ & -88 dBm@no SJ & -75 dBm@-3 dBm SJ \\
\hline Output power & $11 \mathrm{dBm}$ (linear) & $10.4 \mathrm{dBm}$ (linear) & $22 \mathrm{dBm}$ (linear) & $20 \mathrm{dBm}$ (linear) & $22.4 \mathrm{dBm}$ (linear) \\
\hline Phase noise & $\begin{array}{l}-116 \mathrm{dBc} @ 200 \mathrm{kHz} \\
\text {-132dBc@1MHz }\end{array}$ & $\begin{array}{l}\text {-110dBc@200kHz } \\
\text {-127dBc@1MHz }\end{array}$ & $\begin{array}{l}\text {-103dBc@100kHz } \\
\text {-126dBc@1MHz }\end{array}$ & $\begin{array}{l}\text {-104.7dBc@100KHz } \\
\text {-130.6dBc@1MHz }\end{array}$ & $\begin{array}{l}-106.2 \mathrm{dBc} @ 200 \mathrm{kHz} \\
-130.2 \mathrm{dBc} @ 1 \mathrm{MHz}\end{array}$ \\
\hline Power consumption & $1.2 \mathrm{~W}$ & $<276.4 \mathrm{~mW}$ & $660 \mathrm{~mW}$ & $980 \mathrm{~mW}$ & $1.1 \mathrm{~W}$ \\
\hline Die area & $21 \mathrm{~mm}^{2}$ & $18.3 \mathrm{~mm}^{2}$ & $13.5 \mathrm{~mm}^{2}$ & $17.2 \mathrm{~mm}^{2}$ & $16.8 \mathrm{~mm}^{2}$ \\
\hline
\end{tabular}

coupler and antenna and sharing TCXO, protocol controller and data convertor with mobile devices, it can easily be used for portable devices needing UHF RFID applications.

\section{REFERENCES}

[1] K. Finkenzeller, “RFID Handbook,” John Wiley, 2006.

[2] EPCglobal Inc., "EPC C1G2 UHF RFID Protocol for Communications at 860-960MHz,” Version 1.2.0, 2008.

[3] S. Chiu, et al., "A 900MHz UHF RFID Reader Transceiver IC,” IEEE Journal of Solid-State Circuits, Vol. 42, No. 12, 2007, pp. 2822-2833. http://dx.doi.org/10.1109/JSSC.2007.908755

[4] W. T. Wang, et al., "A Single-Chip UHF RFID Reader in $0.18 \mu \mathrm{m}$ CMOS Process,” IEEE Journal of Solid-State Circuits, Vol. 43, No. 8, 2008, pp. 1741-1751. http://dx.doi.org/10.1109/JSSC.2008.925601

[5] L. Ye, et al., "A Single-Chip CMOS UHF RFID Reader
Transceiver for Chinese Mo bile Applications,” IEEE Journal of Solid-State Circuits, Vol. 45, No. 7, 2010, pp. 1316-1327. http://dx.doi.org/10.1109/JSSC.2010.2049459

[6] J. Kim, et al., "A True Single SoC for UHF Mobile RFID Reader,” Proceedings of IEEE ESSCIRC, Helsinki, 12-16 September 2011, pp. 171-174.

[7] MIIT, “800/900MHz RFID Tech. Draft,” No. 205, 2007.

[8] “ISO/IEC RFID 18000-6,” 2nd Edition, December 2010.

[9] ETSI 302 208-1 Technical Requirements and Methods of Measurement, Version 1.1.1, 2004.

[10] J. Y. Jung, et al., “A Novel Carrier Leakage Suppression Front-End for UHF RFID Reader," IEEE Transactions on Microwave Theory and Techniques, Vol. 60, No. 5, 2012, pp. 1468-1477. http://dx.doi.org/10.1109/TMTT.2012.2187675

[11] “MAFRIN0332 Datasheet,” Version2.

[12] A. Safarian, et al., “An Integrated RFID Reader,” Digest of Technological Paper of IEEE ISSCC, San Francisco, 11-15 February 2007, pp. 218-219. 